\title{
Amplified short-wavelength light scattered by relativistic electrons in the laser-induced optical lattice
}

\author{
I. A. Andriyash, ${ }^{1,2, *}$ V. T. Tikhonchuk, ${ }^{3}$ V. Malka, ${ }^{1}$ E. D'Humières, ${ }^{3}$ and Ph. Balcou ${ }^{3}$ \\ ${ }^{1}$ Laboratoire d'Optique Appliquée, ENSTA-ParisTech, CNRS, Ecole Polytechnique, \\ UMR 7639, 91761 Palaiseau, France \\ ${ }^{2}$ P. N. Lebedev Physics Institute, Russian Academy of Sciences, Moscow 119991, Russia \\ ${ }^{3}$ Université de Bordeaux, CNRS, CEA, CELIA, UMR 5107, F33400 Talence, France
}

(Received 19 December 2014; published 29 May 2015)

\begin{abstract}
The scheme of the x-ray free electron laser based on the optical undulator created by two overlapped transverse laser beams is analyzed. A kinetic theoretical description and an ad hoc numerical model are developed to account for the finite energy spread, angular divergence, and the spectral properties of the electron beam in the optical lattice. The theoretical findings are compared to the results of the one- and three-dimensional numerical modeling with the spectral free electron laser code PLARES.
\end{abstract}

DOI: 10.1103/PhysRevSTAB.18.050704

PACS numbers: 41.60.Cr, 42.65.Dr, 52.25.Dg, 41.75.Ht

\section{INTRODUCTION}

The powerful sources of $\mathrm{x}$ rays are now becoming indispensable tools in science, technology, and medicine. Nowadays, high-quality $\mathrm{x}$-ray and extreme ultraviolet (XUV) light is delivered by synchrotron radiation (SR) sources. These installations are typically large scale and include a radio-frequency electron accelerator, combined with an undulator assembled from permanent magnets, and devices for beam manipulation (quadruples, chicanes, steerers, etc.). The SR sources of the last generation-the $\mathrm{x}$-ray free electron lasers (XFELs) - have reached record $G W$ powers for the ångström wavelengths operating in the coherent regime [1]. This regime is provided by the high stability of the beam-undulator interaction, when individually emitting electrons get progressively involved in the process of stimulated scattering and amplify the light in a collective fashion [2].

The wavelength of the radiation produced by an individual electron is $\lambda_{s} \simeq\left(1+K_{0}^{2} / 2\right) \lambda_{u} / 2 \gamma_{e}^{2}$, where $\lambda_{u}$ and $K_{0}$ are the undulator period and its strength parameter, respectively, and $\gamma_{e}$ is the electron Lorentz factor. The parameter $K_{0}$ defines the undulator efficiency, i.e., its capability to deviate the particle transverse momentum. $K_{0}$ is typically proportional to the field amplitude and the period, e.g., for a conventional linear magnetic undulator $K_{0}=0.93 B_{0}[\mathrm{~T}] \lambda_{u}[\mathrm{~cm}]$. Therefore, the maximal x-ray photon energy $2 \pi c \hbar / \lambda_{s}$ is limited by the minimal $\lambda_{u}$ and the energy of the electron. In conventional SR sources, the size of the undulator magnet which provides a sufficiently strong magnetic field is around a centimeter, which makes

\footnotetext{
*igor.andriyash@gmail.com

Published by the American Physical Society under the terms of the Creative Commons Attribution 3.0 License. Further distribution of this work must maintain attribution to the author $(s)$ and the published article's title, journal citation, and DOI.
}

it difficult to reach the sub-ångström radiation wavelengths, requiring larger and more expensive accelerators.

Alternatively, a number of currently explored schemes propose to collide the electron beam with an intense optical laser radiation [3,4]. Such Compton sources can provide a very short, micrometer undulation period with sufficiently large $K_{0}$ values. The rate of the amplification in FELs is typically determined by the parameter $\rho \propto \gamma_{b}^{-1} j_{e}^{1 / 3}\left(K_{0} \lambda_{u}\right)^{2 / 3}$, where $j_{e}=I_{e} / \sigma_{\perp}^{2}$ is the peak flux defined by the current $I_{e}$ and the transverse size of the electron beam $\sigma_{\perp}$, and the beam Lorentz factor $\gamma_{b}$ is defined by the average energy of electrons [5]. Since amplification decreases with $\lambda_{u}$, the efficient operation of the opticalbased XFELs requires a relatively long interaction distance, which imposes severe limitations on the beam quality. As a consequence, while such an idea is being discussed in a number of theoretical works [6-8], any practical realization remains beyond current expectations.

One possible solution to improve the concept of the optical-based XFEL is to replace the conventional rf linac with the laser plasma accelerator (LPA) $[9,10]$. The technology of the LPA has already proved its capability to deliver pico-Coulomb femtosecond beams of $\mathrm{MeV}$ electrons [11] and has now reached the $\mathrm{GeV}$ level $[12,13]$. The incoherent undulator radiation produced by the laser-accelerated electrons was recently observed experimentally $[14,15]$, and the prospects for coherent amplification of this radiation were discussed [16]. Although the laser plasma accelerators now provide the necessary high electron flux, the level of collimation and monoenergeticity required for the x-ray amplification yet remains challenging.

In our work, we tackle this problem by considering a scheme based on a specific configuration of the optical undulator-the optical lattice. The lattice results from the overlap of two identical side laser beams and represents a spatially, transversally modulated electromagnetic field 
structure. The interest for such a structure and its interaction with electron beams originates from the works of Kapitza and Dirac back in 1933 [17] and was later revised in a number of works $[18,19]$. At the same time appeared the idea to use the electron slow motion in the optical lattice as a low-frequency light source $[20,21]$. Recently, we have focused our attention to electron light scattering in the lattice for coherent amplification of short-wavelength radiation [22,23].

A major advantage of the transverse optical lattice is that both it wiggles the electrons in the varying laser field and its spatial modulations act on the electrons via the ponderomotive force, thereby trapping them in the potential channels $[24,25]$. On one hand, such electron guiding prevents electrons from diverging along one direction [26], thus partially preserving the electron flux. On the other hand, potential channels affect the collective behavior of the electrons, predisposing them to a new mechanism of amplification similar to the stimulated Raman scattering [23]. Practically, such a scheme involves the travelingwave technique which has been proposed and discussed in the context of the optical lattice in Ref. [22] and in Refs. [27,28] for the Thomson scattering mechanism. The traveling electromagnetic waves can copropagate with electrons for a long distance and, therefore, may provide a stable amplification.

In the present work, we revise the physics of x-ray amplification in the electron beam trapped in the optical lattice. For this we develop a self-consistent kinetic model in Sec. II and with its help describe the growth of the electromagnetic signal in the analytical approach and by using a simple numerical integration (Sec. III). In Sec. IV, the theoretical results are compared to the threedimensional numerical modeling performed with the unaveraged spectral free electron laser code PLARES [29]. The concluding remarks are given in Sec. V.

\section{THEORETICAL MODEL}

We describe the interaction of the relativistic electrons with the optical lattice and the scattered radiation. The theoretical model is based on the Vlasov kinetic equation and the equation for electromagnetic potential:

$$
\begin{gathered}
\partial_{t} f+(\mathbf{v} \nabla) f+\left(\partial_{t} \mathbf{a}-\mathbf{v} \times \nabla \times \mathbf{a}\right) \partial_{\mathbf{p}} f=0, \\
\left(\partial_{t}^{2}-\nabla^{2}\right) \mathbf{a}=-\int \mathbf{v} f \mathrm{~d} \mathbf{p} .
\end{gathered}
$$

In the present study, we normalize frequencies and wave numbers to $\omega_{0}=2 \pi c / \lambda_{0}$ and $k_{0}=\omega_{0} / c$, where $\lambda_{0}$ is the wavelength of the laser. The distances and time in Eq. (1) are measured in units $c / \omega_{0}$ and $\omega_{0}^{-1}$, the electron velocities and momenta are normalized to the speed of light $c$ and $m_{e} c$, respectively, and the vector potential is in units of $m_{e} c^{2} / e$. In this convention, the electron distribution

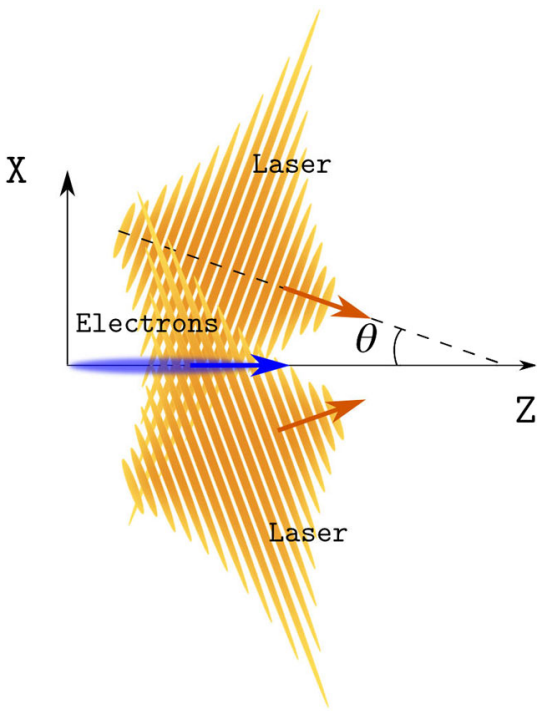

FIG. 1. Optical lattice scheme.

function (EDF) is normalized as $\int f(\mathbf{r}, \mathbf{p}, t) \mathrm{d} \mathbf{p}=n_{e}$, where the electron density $n_{e}$ is in units of its critical value, $n_{c}=\left(m_{e} \omega_{0}^{2} / 4 \pi e^{2}\right)$.

The beam of electrons travels at a relativistic velocity $v_{b}=\sqrt{1-\gamma_{b}^{-2}} \simeq 1$ in the overlap of two identical laser pulses incident symmetrically with the angle $\theta$ (see Fig. 1). The vector potential $\mathbf{a}=\mathbf{a}_{L}+\mathbf{a}_{s}$ in Eq. (1) accounts for the lattice field

$$
\mathbf{a}_{L}=2 a_{0} \mathbf{e}_{y} \sin (x \sin \theta) \cos (t-z \cos \theta),
$$

and the signal wave $\mathbf{a}_{s}$, associated with the scattered radiation. This signal copropagates with electrons:

$$
\mathbf{a}_{s}=a_{s} \mathbf{e}_{y} \cos \left[k_{s}(t-z)\right],
$$

and its amplitude $a_{s}$ is assumed to be small, so in our analysis we retain only linear terms on $a_{s}$. Note that for an electron propagating along the $z$ axis the undulation period provided by the lattice field is

$$
\lambda_{u}=\lambda_{0} /\left(1-v_{b} \cos \theta\right) .
$$

One approximation commonly used to describe the motion of charged particles in a strong laser field is to divide this motion into "fast" and "slow" parts. The fast reaction of an electron is to follow oscillations of the electromagnetic field directly. On the other hand, the particles are also driven by the period-averaged ponderomotive force, defined by the field spatial gradients. The optical lattice field in Eq. (2a) has the transverse gradient, which creates the ponderomotive force along the $x$ axis:

$$
\mathbf{F}^{(0)}=-\mathbf{e}_{x} \gamma_{b}^{-1} a_{0}^{2} \partial_{x}|\sin (x \sin \theta)|^{2}
$$


and produces a series of potential channels with widths $L_{x}=\pi / 2 \sin \theta$.

In the presence of the signal wave (2b), its interference with the lattice produces a beat wave in the longitudinal direction. This wave acts on the electrons via the ponderomotive force:

$$
\begin{aligned}
\mathbf{F}^{(1)}= & \mathbf{e}_{z} a_{0} a_{s} \gamma_{b}\left[k_{s}\left(1-v_{b}\right)+v_{b}-\cos \theta\right] \sin (x \sin \theta) \\
& \times \sin \left[\left(1-k_{s}\right) t-\left(\cos \theta-k_{s}\right) z\right] .
\end{aligned}
$$

which is calculated in the reference frame of the electron beam (cf. [30]) and then translated to the laboratory system.

\section{A. Unperturbed beam in the lattice}

The unperturbed state of the electron beam trapped in the lattice potential is defined only by the transverse ponderomotive force Eq. (4a). The characteristics of the kinetic equation (1a) in this case can be written as

$$
\frac{\mathrm{d} x}{v_{x}}=\frac{\mathrm{d} p_{x}}{F^{(0)}}, \quad \mathrm{d} t=\frac{\mathrm{d} z}{v_{z}},
$$

and they define the particle trajectories, along which the initial EDF remains constant. The first equation of Eq. (5) represents the energy conservation condition

$$
p_{x}^{2} / 2 a_{0}^{2}+\sin ^{2}(x \sin \theta)=\xi^{2},
$$

and, neglecting the variations of the electron Lorentz factor, we may calculate the orbits of the electrons as

$$
\sin (x \sin \theta)=\operatorname{sn}\left(\xi \Omega_{0} t+\Theta ; \xi^{-2}\right),
$$

where $\operatorname{sn}(u, m) \equiv \sin (\phi)$ is a Jacobi elliptic function defined as the inverse of elliptic integral $u=$ $\int_{0}^{\phi}\left(1-m \sin ^{2} \theta\right)^{-1 / 2} \mathrm{~d} \theta$. Here we have also denoted a normalized excursion $\xi$, and $\Omega_{0}=\sqrt{2} a_{0} \sin \theta / \gamma_{b}$ is a frequency of the small-amplitude oscillations. The phase $\Theta$ is defined by the initial coordinate and momentum of the particle.

If $\xi<1$, the electron is trapped, and it oscillates in a channel of the optical lattice with the frequency

$$
\Omega=\frac{\pi \Omega_{0}}{2 K(\xi)} \simeq \Omega_{0}\left(\frac{\sin \pi \xi}{\pi \xi}\right)^{1 / 6} \approx \Omega_{0}\left(1-\xi^{2} / 3\right)
$$

where $K(m)=\int_{0}^{\pi / 2}\left(1-m \sin ^{2} \phi\right)^{-1 / 2} \mathrm{~d} \phi$ is the quarter period of the elliptic integral.

The first approximation in Eq. (8) was previously presented in Ref. [26], and its accuracy is better than $99 \%$ for $\xi<0.999$. The alternative expression in the leftmost part of Eq. (8) is derived by expanding $\sin ^{2}(x \sin \theta)$ into the series, and it retains a $98 \%$ accuracy for $\xi<0.85$. The latter is more convenient for analytical developments, and we will use it further with the electron orbits Eq. (7) approximated by sinusoids. Finally, the set of electron trajectories in the $(x, z)$ phase plane will read

$$
\begin{aligned}
X & =\left[x_{0} \cos (\Omega t)+p_{x 0} /\left(\Omega \gamma_{b}\right) \sin (\Omega t)\right], \\
P_{x} & =\left[\left(p_{x 0} \cos (\Omega t)-x_{0} \Omega \gamma_{b} \sin (\Omega t)\right],\right. \\
Z & =z_{0}+v_{z} t, \quad v_{z}=\mathrm{const} .
\end{aligned}
$$

For a simple analytical model, one can assume homogeneous electron distributions in the transverse phase plane, where the particles are trapped, and in the longitudinal direction, which is not affected by the lattice field. The electron distribution function in this case reads

$$
f=\frac{n_{0}}{4 \sqrt{2} a_{0} \xi_{0} \delta \gamma_{b}} \eta\left(\xi_{0}^{2}-\xi^{2}\right) \eta\left(\delta \gamma_{b}-\left|p_{z}-p_{b}\right|\right),
$$

where $\eta(x)$ is a unit-step function, $n_{0}=n_{e}(x=0)$ is a maximal electron density, and $\delta \gamma$ is a longitudinal energy spread of electrons. The maximal excursion is limited by the potential scale $\xi_{0} \leq 1$.

\section{B. Interaction of the electron density perturbations and the signal electromagnetic wave}

The force (4b) associated with the signal wave acts on the particles and rearranges their longitudinal positions, thus generating the perturbation of $\operatorname{EDF} f \rightarrow f+\delta f$. In turn, this perturbation acts back on the signal field through the electron current along the $y$ axis in the right-hand side of Eq. (1b). Such coupling may provide a resonant interaction between electron and electromagnetic modes resulting in their amplification or damping similarly to the stimulated Raman scattering [31].

During the early, linear stage of interaction, the amplitude of the signal wave and the EDF perturbations are small $a_{s} \ll a_{0}, \delta f \ll f$. The current $\int \mathbf{v} f \mathrm{~d} \mathbf{p}$, associated with the resonant interaction in Eq. (1b), includes the generating term $a_{L} \delta n / \gamma_{b}$ and the term $a_{s} n_{e} / \gamma_{b}$, which is responsible for the dispersion of the signal resulting from its interaction with the space-charge plasma waves. In the simplest case, we may neglect this dispersion as well as the diffraction of the amplified wave, therefore, assuming a plane transverse profile. Keeping only the first-order resonant perturbation terms, we write the signal wave equation as

$$
\partial_{t} a_{s}=i \frac{a_{0} n^{*}}{\gamma_{b} k_{s}} \sin (x \sin \theta),
$$

where $n^{*}$ is related to the electron density perturbations as

$$
\delta n=\int \delta f \mathrm{~d} \mathbf{p}=\operatorname{Re}\left[n^{*} \mathrm{e}^{i\left(\cos \theta-k_{s}\right) z-i\left(1-k_{s}\right) t}\right] .
$$

To study the interaction of an electromagnetic field with relativistic electrons, it is convenient to introduce the 
coordinates which follow the center of the electron beam $z \rightarrow z+v_{b} t$. The Doppler-shifted frequencies of the lattice and the signal wave are $\tilde{\omega}_{e}=\left(1-v_{b} \cos \theta\right)$ and $\tilde{\omega}_{s}=$ $\left(1-v_{b}\right) k_{s}$, respectively, and the interaction is resonant when $\tilde{\omega}_{e} \approx \tilde{\omega}_{s}$ (we will discuss the resonance condition in detail in Sec. III B). The ponderomotive force Eq. (4b) and the density perturbations in the moving coordinate system read

$$
\begin{aligned}
F^{(1)} & =\frac{a_{0} a_{s} k_{s}}{\gamma_{b}} \sin (x \sin \theta) \sin \left[\Omega_{s} t+k_{s} z\right], \\
\delta n & =\operatorname{Re}\left[n^{*} \mathrm{e}^{-i\left(\Omega_{s} t+k_{s} z\right)}\right],
\end{aligned}
$$

where we have assumed $k_{s} \gg \cos \theta$, and $\Omega_{s}=\tilde{\omega}_{e}-\tilde{\omega}_{s}$ is a frequency of the slow oscillations of longitudinal ponderomotive force. Note that it is the force $F^{(1)}$ which produces the modulations of electron density-the bunched structure. These modulations propagate with the velocity $-\Omega_{s} / k_{s}$, and the sign of $\Omega_{s}$ defines the propagation direction. In the case of $\Omega_{s}<0$, the modulations copropagate with the electron beam and accelerate the particles, thereby draining the energy of the electromagnetic field. Otherwise, if the signal is downshifted, $\tilde{\omega}_{s}<\tilde{\omega}_{e}$, the electrons give the energy to the wave amplifying it. In what follows, we will consider only this growing mode, always assuming $\Omega_{s}>0$.

Let us describe the dynamics of $n^{*}$ by perturbing the kinetic equation (1b) and averaging it over the lattice field period:

$$
\left[\partial_{t}+v_{x} \partial_{x}+v_{z} \partial_{z}+F^{(0)} \partial_{p_{x}}\right] \delta f=-F^{(1)} \partial_{p_{z}} f,
$$

where only the first-order terms are retained. The operator in the left-hand side is simply the time derivative $\mathrm{d} \delta f / \mathrm{d} t$ calculated along the trajectories (9). Substituting these trajectories into Eq. (13) and considering the model EDF Eq. (10), the integration of Eq. (13) over the momenta may be simplified as

$$
n^{*}=\frac{a_{0} n_{0} k_{s}^{2}}{4 \gamma_{b}^{4} \xi_{0}} \sin \chi \int_{-\infty}^{t} \mathrm{~d} t^{\prime} a_{s}\left(t^{\prime}\right) G\left(t^{\prime}-t\right)
$$

where we denote $\chi=x \sin \theta$ and the function

$$
G(t)=\frac{\sin \left(k_{s} \delta v_{z} t\right)}{k_{s} \delta v_{z}} \int_{0}^{\xi_{0}^{2}} \mathrm{~d} \xi^{2} \frac{\mathrm{e}^{i\left(\Omega-\Omega_{s}\right) t}}{\sqrt{\xi^{2}-\sin ^{2} \chi}}
$$

describes the dynamics of the electron density perturbations driven by the ponderomotive forces. The longitudinal energy spread of electrons is represented here by the parameter $\delta v_{z}=\delta \gamma / \gamma_{b}^{3}$. As we have mentioned before, the nonresonant upshifted mode $\exp \left[-i\left(\Omega+\Omega_{s}\right) t\right]$ is neglected.

\section{ANALYSIS OF THE STIMULATED SCATTERING}

Equations (11) and (14) describe the evolution of the coupled electron and electromagnetic perturbations. The conditions, at which the signal wave may be amplified, are defined by the dispersion properties of the electron beam mode. For simplicity, in the following analysis, we will assume that electrons fill completely the lattice channel, so that $\xi_{0}=1$.

In our model, the electron mode is described by the function $G(t)$ in Eq. (14). If we neglect the dependence of $\Omega$ on $\xi$, the electron mode would be harmonic and the integral in the function $G$ may be simplified as

$$
B=\int_{0}^{1} \frac{\mathrm{e}^{i \Omega_{0} t} \mathrm{~d} \xi^{2}}{\sqrt{\xi^{2}-\sin ^{2} \chi}}=\mathrm{e}^{i \Omega_{0} t} \cos \chi
$$

The dependence of $\Omega$ on $\xi$ results in a deformation of the electron mode with respect to the $x$ coordinate. Considering the second approximation in Eq. (8), one may qualitatively expect that the electron mode spectrum after averaging will depend on $x$ as $B \propto \mathrm{e}^{i \Omega^{\prime} t}$, where $\Omega^{\prime}=\Omega_{0}\left(1-\sin ^{2} \chi / 3\right)$. More accurately, the mode can be calculated as

$$
B=\sqrt{6 \pi / t}[C(\alpha)-i S(\alpha)] \mathrm{e}^{i \Omega^{\prime} t},
$$

where $S(\alpha)$ and $C(\alpha)$ are the Fresnel integrals and $\alpha=\cos \chi \sqrt{2 t / 3 \pi}$.

The numerically calculated spectral-spatial map of the electron beam modes given by Eqs. (15) and (16) is shown in Fig. 2. The deformated electron mode Eq. (16) is shown in red, and it corresponds to a wider spectral region, where the signal wave interacts resonantly with the electrons.

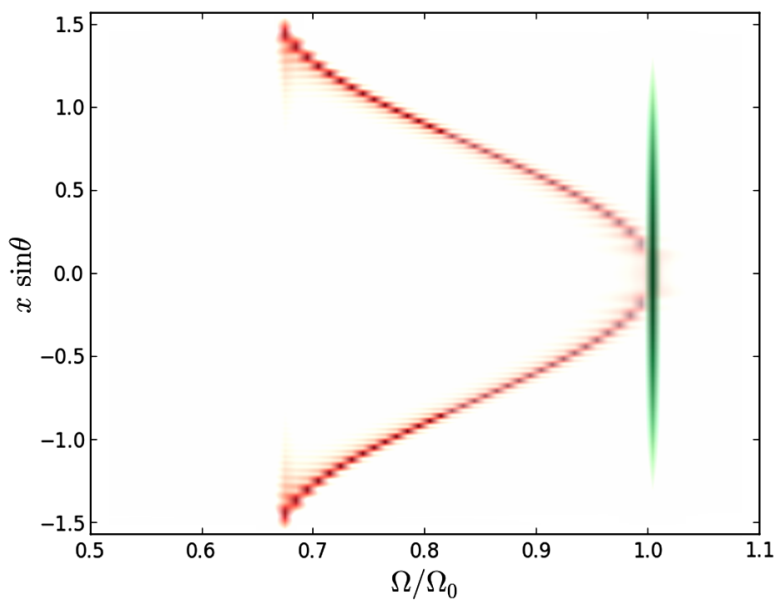

FIG. 2. Electron beam mode frequency as a function of the $x$ coordinate for the elliptic (red) and the harmonic (green) electron orbits. 


\section{A. Interaction parameters}

For the following analysis, let us consider the optical lattice field which is produced by two plane waves corresponding to the pulses of a Ti:sapphire laser with the wavelength $\lambda_{0}=800 \mu \mathrm{m}$ and the intensity $2.16 \times 10^{16} \mathrm{~W} / \mathrm{cm}^{2}$. The incidence angles of the laser waves to the electron propagation direction are chosen to be $\theta=10^{\circ}$.

We assume a $100 \mathrm{pC}$ beam of electrons with moderate energies of $40 \mathrm{MeV}$, which can be delivered either by a laser plasma accelerator or by a compact linear accelerator. The scheme with LPA electrons is all-optical and, thus, can be driven by a single laser system. Such a design is advantageous for synchronization of the traveling optical lattice with the electron beam, and at the same time it allows one to place the lattice at a short, millimeter distance from the accelerator. In this case, one can benefit from the initially high flux and the small size of the electron beam and avoid beam transport issues typical for LPA [32]. Moreover, such a scheme can be conveniently complemented with the plasma lens technique, which has recently shown the capability to significantly reduce the electron beam divergence [33]. Alternatively, a compact linac with a photocathode gun can provide an electron beam with the low emittance and energy spread [34]. The stability and quality of the linac-produced electrons give the advantage to such sources, however, the time jitter in such a design may present a challenge for the lattice-beam synchronization.

For the numerical tests, the electron beam can be considered as a homogeneous cylinder with the corresponding duration $3.75 \lambda_{0} / c$ and the radius $R_{b}=1.3 \lambda_{0}$, which fits the width of the lattice channel. The initial spread of the electron transverse momenta is chosen to also fit the trapping condition, $\delta p_{x}=\sqrt{2} a_{0}$. In physical units, this corresponds to $1 \mathrm{mrad}$ of $\mathrm{rms}$ angular divergence or normalized emittance $\epsilon_{x}=4 \mathrm{~mm} \mathrm{mrad}$, and the divergence along the nontrapped $y$ direction can be assumed to be lower- $0.36 \mathrm{mrad}$, which corresponds to $\epsilon_{y}=$ $1.5 \mathrm{~mm} \mathrm{mrad}$. The spread of electron energies in our tests varies from the monoenergetic case $\delta \gamma=0$ to a more realistic one $\delta \gamma / \gamma_{b}=0.03$. In dimensionless units, the chosen electron density and laser amplitude are $n_{0}=$ 0.0352 and $a_{0}=0.1$, respectively. Such parameters are closer to the LPA electrons, but the model can be applied to the case of a linac beam.

Note that in the present analysis we do not consider the effects of the electrostatic fields, which act to inhibit the development of electron density modulations. Partially, this is justified by the fact that the Coulomb repelling for the relativistic beams decreases with the electron relativistic factor $\gamma_{b}$. Nevertheless, for a sufficiently long interaction time these effects may become important. A qualitative way to estimate the importance of the spacecharge effects is to compare the wavelength of plasma oscillations, $\lambda_{p}=\sqrt{\gamma_{b}^{3} / n_{0}}$, with the typical interaction scales. For the chosen parameters $\lambda_{u} / \lambda_{p} \simeq 10^{-2}$, which means that the space-charge field does not affect the beam during a single electron oscillation. In the following section, we consider such a comparison with the gain length, while the rigorous account for the space-charge fields we leave for future studies.

\section{B. Harmonic electron mode}

Let us first study amplification for the case of the harmonic electron mode Eq. (15), assuming all electrons oscillating with the same frequency $\Omega_{0}$. In this case, Eqs. (11) and (14) may be linearized analytically with the help of the Laplace transform or by reducing Eq. (14) to the differential form and performing the time-domain Fourier transform:

$\left[\left(\omega-\Omega_{s}+\Omega_{0}\right)^{2}-k_{s}^{2} \delta v_{z}^{2}\right]\left\langle n^{*}\right\rangle_{\omega}=\left\langle a_{s}\right\rangle_{\omega} \frac{a_{0} n_{0} k_{s}^{2}}{2 \gamma_{b}^{4}} \sin \chi \cos \chi$.

The Fourier component $\left\langle a_{s}\right\rangle_{\omega}$ can be found from Eq. (11), and substituting it to Eq. (17) we obtain the dispersion equation:

$$
\omega\left[\left(\omega-\Omega_{s}+\Omega_{0}\right)^{2}-k_{s}^{2} \delta v_{z}^{2}\right]=\frac{a_{0}^{2} n_{0} \tilde{\omega}_{e}}{\gamma_{b}^{3}} \sin ^{2} \chi \cos \chi .
$$

The dependence on the transverse coordinate in the righthand side of Eq. (18) corresponds to the modulation of a coupling between the electron and electromagnetic modes. Physically, this means that the signal wave interacts with electrons more efficiently around the coupling maxima $\chi= \pm 1 / \sqrt{3}$, which tends to produce an amplified wave with a profile modulated along the $x$ axis. For the onedimensional analysis, we consider an effective mode coupling by averaging $\left\langle\sin ^{2} \chi \cos \chi\right\rangle=2 / 3 \pi$.

The coupling of the electromagnetic and electron modes in Eq. (18) allows a resonant energy transfer from the particles to the electromagnetic field. If the solution $\omega$ has a negative imaginary part, it corresponds to the modes which grow exponentially. It is easy to see that, for a monoenergetic beam with $\delta \gamma=0$, the maximal growth occurs for $\Omega_{s}=\Omega_{0}$, and it defines the FEL parameter [5]:

$$
\rho_{0}=\left(\frac{a_{0}^{2} n_{0}}{12 \pi \gamma_{b}^{3}(1-\cos \theta)^{2}}\right)^{1 / 3},
$$

which corresponds to the power gain length $L_{g 0}=$ $\lambda_{u} /(4 \pi \sqrt{3} \rho)$.

The finite electron energy spread strongly decreases the amplification rate and modifies its spectral structure by narrowing the resonance bandwidth and downshifting the central wavelength as 


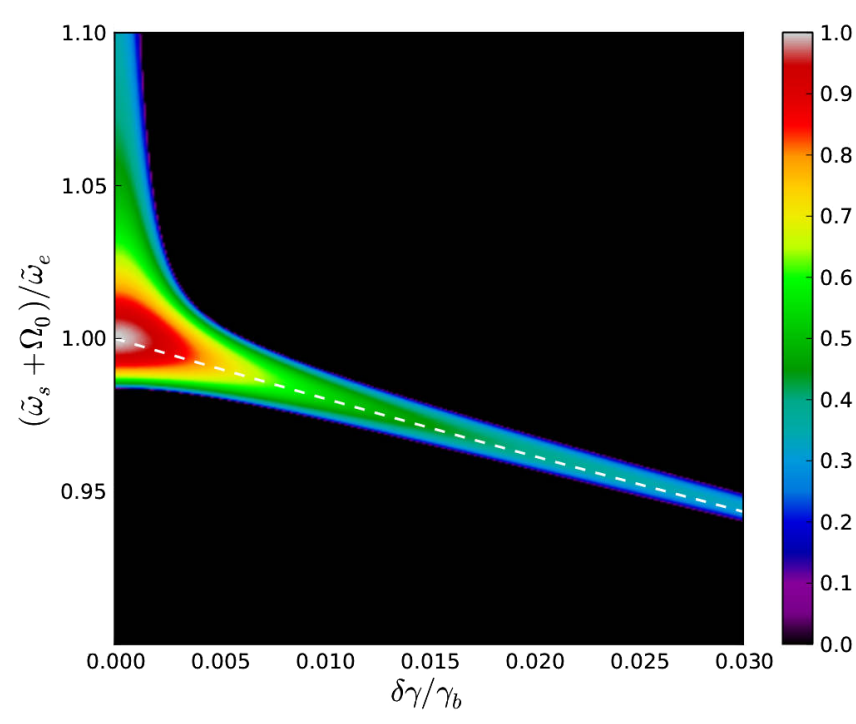

FIG. 3. Normalized FEL parameter $\rho / \rho_{0}$ as a function of longitudinal temperature and signal frequency given by Eq. (18). The white dashed line corresponds to the resonance condition Eq. (20).

$$
\omega_{s} \approx 2 \gamma_{b}^{2}\left(\tilde{\omega}_{e}-\Omega_{0}\right) /\left(1+2 \delta \gamma / \gamma_{b}\right) .
$$

The maximal FEL parameter in this case decreases with the energy spread, and when $\delta \gamma / \gamma_{b} \gtrsim \rho_{0}$, it can be estimated as $\rho \approx \sqrt{2 / 3}\left(\delta \gamma / \gamma_{b}\right)^{-1 / 2} \rho_{0}^{3 / 2}$.

The dependence of the FEL parameter on the energy spread and signal frequency is presented in Fig. 3, where the resonance condition Eq. (20) is shown with the dashed white line. The maximal FEL parameter corresponding to the chosen interaction parameters is $\rho_{0}=4.38 \times 10^{-3}$, and this defines the gain length $L_{g} \simeq 10 \lambda_{u}$. Comparing this value with the plasma wavelength, we find that $L_{g} / \lambda_{p} \simeq 0.18$, which allows us to consider that the space-charge fields produce only a second-order effect for the linear amplification.

\section{Account for the ellipticity of electron orbits}

For a more accurate analysis, one has to account for the dependence of electron oscillation frequency on the excursion parameter $\xi$, which may be approximately described by Eq. (16). In this case, the analytical linearization of Eqs. (11) and (14) would require further approximations and affect the accuracy of such a description. Alternatively, these equations may be solved numerically on a finite time interval via iterations. For this, we write Eqs. (11) and (14) for the normalized functions $N^{(n)}=a_{0} n^{*} /\left(2 \gamma_{b}^{3} \tilde{\omega}_{s} \tilde{\omega}_{e}\right)$ and $A^{(n)}=a_{s} / a_{0}$ in the following form:

$$
\begin{gathered}
N^{(n)}=-12 \pi \rho_{0}^{3} w \sin \chi \int_{0}^{\tau} \mathrm{d} \tau^{\prime} A^{(n)}\left(\tau^{\prime}\right) \tilde{G}\left(\tau-\tau^{\prime}\right), \\
A^{(n+1)}=\frac{i}{\pi} \int_{0}^{\tau} \mathrm{d} \tau^{\prime} \int_{-\pi / 2}^{\pi / 2} \mathrm{~d} \chi N^{(n)},
\end{gathered}
$$

where $\tau=\tilde{\omega}_{e} t, w=\tilde{\omega}_{s} / \tilde{\omega}_{e}, O=\Omega_{0} / \tilde{\omega}_{e}$,

$$
\tilde{G}=\frac{\sin w \delta g(\tau)}{w \delta g} \mathrm{e}^{i(1-w) \tau} B(O, \tau, \chi),
$$

$\delta g=2 \delta \gamma / \gamma_{b}$, and the function $B$ is defined by Eq. (16). Considering a small arbitrary "seed" $A^{(0)}=$ const, we calculate the corresponding $N^{(0)}$ over the time interval $\left(0, \tau_{0}\right)$ from Eq. (21a) and then integrate Eq. (21b) to obtain $A^{(1)}$. The solution to Eq. (21) can be obtained by consequently repeating this procedure till the system converges: $\left\|A^{(n)}-A^{(n-1)}\right\| \ll\left\|A^{(n)}\right\|$.

In practice, this algorithm converges rapidly and can be efficiently used for parametric studies with complicated electron modes. Let us test the numerical model for the case of the elliptic electron mode (16). In Fig. 4(a), we plot the evolution of the signal wave amplitude depending on the frequency for the same parameters as in Fig. 3 and the energy spread $\delta \gamma / \gamma_{b}=0.01$. As expected, the amplified mode grows exponentially and is downshifted due to the electron thermal motion. One can also see excitation of the nonresonant mode upshifted due to the electron thermal motion.

In Fig. 4(b), we reproduce numerically the map of the FEL parameter as a function of the frequency and energy spread for the same parameters as in Fig. 3. One may see a remarkable agreement in Figs. 3 and 4(b) in most of the features, and the maximal FEL parameter $\rho=4.21 \times 10^{-3}$ in this calculation agrees with $\rho_{0}$ given by Eq. (19). The feature observed along

$$
\tilde{\omega}_{s} \simeq\left(\tilde{\omega}_{e}-\Omega_{0}\right) /\left(1-2 \delta \gamma / \gamma_{b}\right)
$$

corresponds to a nonresonant upshifted electron mode, and it is shown with the white dashed curve in Fig. 4(b). The center of the resonant region is slightly shifted, which results from the fact that the frequency of elliptic electron oscillations $\Omega^{\prime}$ averaged over the distribution is lower than $\Omega_{0}$.

This model also allows one to include the nonlinear effects, e.g., the divergence of the electron beam along the nontrapped direction or the dynamics of the lattice amplitude. In the present analysis, we account for the electron beam divergence by adding the factor

$$
n_{0}(t) / n_{0}(0)=\left[1+\left(\delta v_{y} t / \sigma_{y}\right)^{2}\right]^{-1 / 2}
$$

to the integral in Eq. (21a). This factor describes a progressive decrease of electron density caused by the divergence and will be used later when comparing with the three-dimensional numerical tests. 
(a)

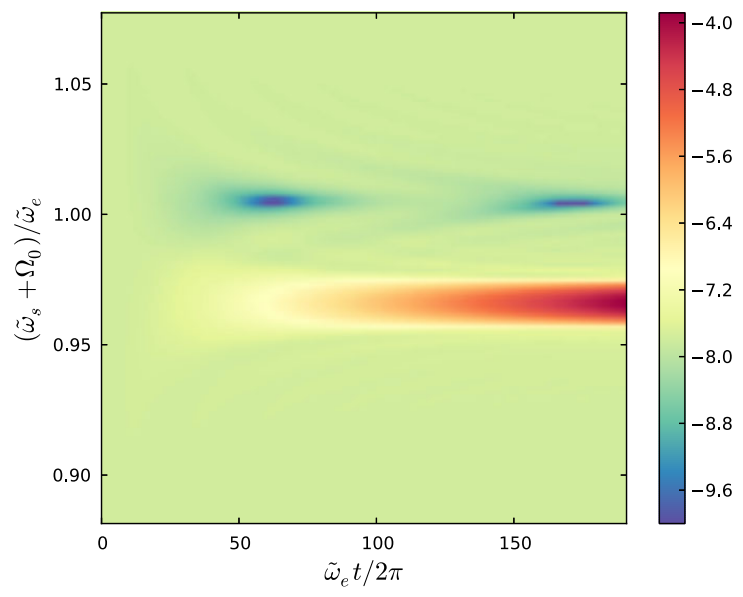

(b)

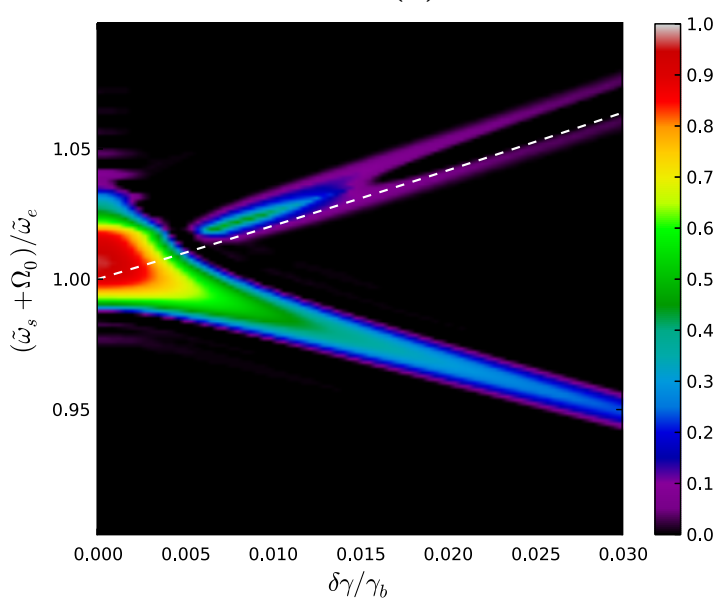

FIG. 4. (a) Temporal dynamics of the signal wave as a function of its frequency calculated numerically. The color scale is logarithmic. (b) Normalized FEL parameter $\rho / \rho_{0}$ as a function of the longitudinal temperature and the signal frequency calculated numerically for the elliptic electron mode.

\section{SIMULATIONS WITH THE FREE ELECTRON LASER CODE}

The theoretical model developed in the previous section describes the amplification at the linear stage, when the main electron distribution is not affected by the growth of electron density perturbations. Therefore, such a model cannot describe the instability saturation, which occurs when a significant part of the electrons are trapped in the longitudinal potentials and stop transferring energy to the signal wave. Near the saturation, the resonance properties of the electron beam mode (e.g., electron energy spread) may also be significantly modified.

To account for the nonlinear effects, a self-consistent description is required. For this, we use the free electron laser code PLARES [29]. In this code, the relativistic electrons are presented by the macroparticles, and their unaveraged three-dimensional motion is coupled with the electromagnetic field calculated with the spectral Maxwell solver. The code may operate in the Cartesian or axisymmetric geometries, and for the present study we use the Cartesian solver, which allows one to model the asymmetric radiation profiles.

The general parameters in the numerical simulations are described in Sec. III A. In addition, for a simple model of the injection, we consider that electrons first enter a $300 \lambda_{0}=4.5 \lambda_{u}$ region, where the lattice field amplitude grows linearly along the $z$ axis before reaching its maximal value $a_{0}=0.1$, which then remains constant during the simulation. The shot noise in the system is completely suppressed, and, to avoid effects of the spontaneous emission amplification, the simulations are seeded at the resonant wavelength of $4.4 \mathrm{~nm}$ with a $10 \mathrm{~kW}$ pulse. In the three-dimensional case, the transverse profiles of the seed are Gaussian and correspond to $1.7 \mu \mathrm{m}$ waist.
Let us first present the steady-state simulations, where the radiation field is considered as a single-frequency infinitely long wave and it interacts with a small fraction of the electron beam assuming the periodic boundary conditions. This case is close to the theoretical description developed in Sec. III; however, it accounts for the dynamics of the electron distribution and includes all related nonlinear effects. The results for the case of the monoenergetic electron beam in one-dimensional (blue solid curve) and three-dimensional (green solid curve) simulations are presented in Fig. 5. The results of PLARES simulations are compared with the description provided by the ad hoc model (21) (dashed curves), where the three-dimensional model also accounts for the beam divergence equation (22). For the one-dimensional case, we see a good agreement for

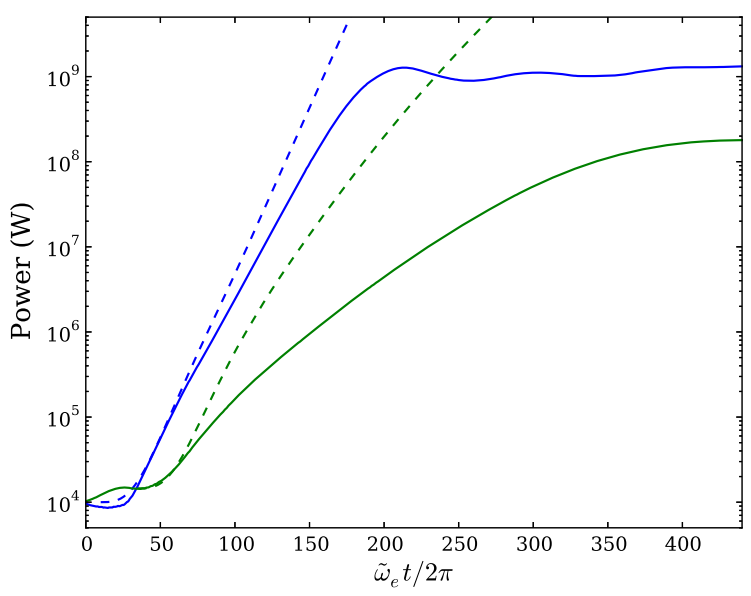

FIG. 5. Steady-state simulations. Radiation power as a function of time for the one-dimensional (blue lines) and threedimensional (green lines) cases modeled by PLARES (solid lines) and with help of ad hoc iterative model (21) (dashed lines). 


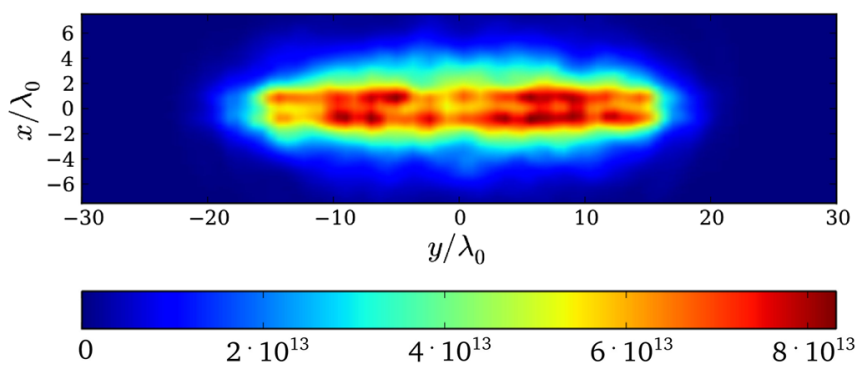

FIG. 6. Steady-state simulations. The intensity profile of the $\mathrm{x}$ rays at the end of the interaction in three-dimensional simulation. The color scale is in $\mathrm{W} / \mathrm{cm}^{2}$.

the early stage of amplification, while during the main stage the growth rate is reduced by approximately $15 \%$ due to the modification of the electron beam distribution function. In the three-dimensional simulation, the amplification decreases even more due to the signal wave diffraction. The saturation occurs at the distances $L_{\mathrm{sat}}=$ 1 and $2 \mathrm{~cm}$ and reaches relatively high powers at the level of $1 \mathrm{GW}$ and $200 \mathrm{MW}$ for the 1D and 3D simulations, respectively.

The transverse profile of the radiation at the end of the 3D simulation is shown in Fig. 6. One may clearly observe that the resulting signal is stretched along the nontrapped direction due to the divergence of the electron beam and is also affected by the diffraction. The intensity modulations along the $x$ axis correspond to the $\chi$ dependence of coupling between modes in Eq. (18).

To study how the amplification is affected by electron energy spread, we run a series of one-dimensional timedependent simulations. The "time dependence" here means

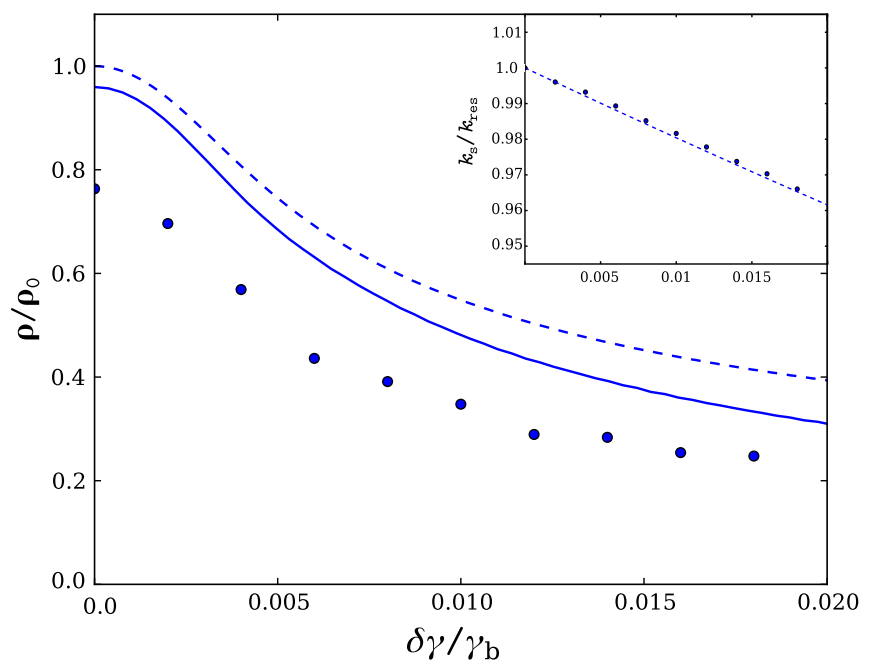

FIG. 7. Dependence of the FEL parameter on the energy spread of the electrons for the analytical (dashed line) and numerical ad hoc (solid line) models (21) and in the PLARES simulations (dots). The inset shows the dependence of the normalized resonant wave number on the energy spread in the simulations (dots) and according to condition (20) (dashed line). that the spectral dynamics of the signal wave is modeled, and the longitudinal profiles of the electron beam and of the seeding pulse are considered. In this model, the resonance condition appears naturally corresponding to the dominant mode. The 1.5 fs seed pulse with the Gaussian longitudinal profile is used in the simulation, and its length is chosen to be much shorter than the length of the electron beam.

In Fig. 7, we plot the FEL parameter as a function of $\delta \gamma / \gamma_{b}$ for the analytical model (dashed curve), ad hoc numerical model (solid curve) and PLARES simulations (dots). The results indicate a good qualitative agreement in the instability behavior and demonstrate the quantitative difference of about $20 \%$ in the values, which is also observed in Fig. 5. The central wave numbers corresponding to the growing mode in the simulations are shown in the inset in Fig. 7 (dots), and it confirms that the resonance follows the condition (20), shown with a dashed line.

The results presented in this section prove the good quality of the theoretical model. The cases of more complex electron distributions can be considered in the same way. The three-dimensional analysis indicates the importance of the divergence of electron beam and the diffraction of the amplified radiation.

\section{SUMMARY}

We have presented a kinetic theory of the linear regime of the $\mathrm{x}$-ray or XUV amplification in the electron beam, which travels in a laser-induced transverse optical lattice. The theory accounts for the energy spread and angular divergence of electron beam. For a simple realistic model of electron distribution, the analytical expressions describe the amplification rate and its dependence on the longitudinal energy spread of electrons. This point has a crucial importance for the practical realizations of such a SR source. The presented results are confirmed by numerical simulations with the spectral FEL code PLARES, where the electron distribution is modeled via particle methods and the dynamics of electromagnetic field is modeled in a consistent way.

The presented analysis indicates that the amplification of the XUV light in the optical lattice is possible for electron parameters typical of the state-of-the-art laser plasma accelerators. The account for the three-dimensional effects evidences that the electron guiding in the optical lattice plays an important role by preserving the electron flux. This opens a path for practical realizations of the optical lattice XFEL without additional components for electron focusing.

\section{ACKNOWLEDGMENTS}

This work was partially supported by the Agence Nationale de la Recherche (ANR) through the LUCEL$\mathrm{X}$ Project (ANR-13-B504-011), and by the European Research Council through the X-Five ERC project (Contract No. 339128). 
[1] P. Emma et al., Nat. Photonics 4, 641 (2010).

[2] B. W. J. McNeil and N. R. Thompson, Nat. Photonics 4, 814 (2010).

[3] F. Albert et al., Phys. Rev. ST Accel. Beams 13, 070704 (2010).

[4] K. Ta Phuoc, S. Corde, C. Thaury, V. Malka, A. Tafzi, R. C. Shah, S. Sebban, and A. Rousse, Nat. Photonics 6, 308 (2012).

[5] Z. Huang and K.-J. Kim, Phys. Rev. ST Accel. Beams 10, 034801 (2007).

[6] P. Sprangle and A. T. Drobot, J. Appl. Phys. 50, 2652 (1979).

[7] A. Bacci, M. Ferrario, C. Maroli, V. Petrillo, and L. Serafini, Phys. Rev. ST Accel. Beams 9, 060704 (2006).

[8] P. Sprangle, B. Hafizi, and J. R. Peñano, Phys. Rev. ST Accel. Beams 12, 050702 (2009).

[9] E. Esarey, C. B. Schroeder, and W. P. Leemans, Rev. Mod. Phys. 81, 1229 (2009).

[10] V. Malka, Phys. Plasmas 19, 055501 (2012).

[11] J. Faure, C. Rechatin, A. Norlin, A. Lifschitz, Y. Glinec, and V. Malka, Nature (London) 444, 737 (2006).

[12] H. T. Kim, K. H. Pae, H. J. Cha, I. J. Kim, T. J. Yu, J. H. Sung, S. K. Lee, T. M. Jeong, and J. Lee, Phys. Rev. Lett. 111, 165002 (2013).

[13] W. P. Leemans, A. J. Gonsalves, H.-S. Mao, K. Nakamura, C. Benedetti, C. B. Schroeder, Cs. Tóth, J. Daniels, D. E. Mittelberger, S. S. Bulanov, J.-L. Vay, C. G. R. Geddes, and E. Esarey, Phys. Rev. Lett. 113, 245002 (2014).

[14] H.-P. Schlenvoigt et al., Nat. Phys. 4, 130 (2008).

[15] M. Fuchs et al., Nat. Phys. 5, 826 (2009).

[16] K. Nakajima, Nat. Phys. 4, 92 (2008).

[17] P. L. Kapitza and P. A. M. Dirac, Math. Proc. Cambridge Philos. Soc. 29, 297 (1933).

[18] P. H. Bucksbaum, D. W. Schumacher, and M. Bashkansky, Phys. Rev. Lett. 61, 1182 (1988).

[19] D. L. Freimund, K. Aflatooni, and H. Batelaan, Nature (London) 413, 142 (2001).
[20] M. V. Fedorov, K. B. Oganesyan, and A. M. Prokhorov, Appl. Phys. Lett. 53, 353 (1988).

[21] S. Sepke, Y. Y. Lau, J. P. Holloway, and D. Umstadter, Phys. Rev. E 72, 026501 (2005).

[22] Ph. Balcou, Eur. Phys. J. D 59, 525 (2010).

[23] I. A. Andriyash, E. d'Humières, V. T. Tikhonchuk, and Ph. Balcou, Phys. Rev. Lett. 109, 244802 (2012).

[24] I. A. Andriyash, Ph. Balcou, and V. T. Tikhonchuk, Eur. Phys. J. D 65, 533 (2011).

[25] E. N. Frolov, A. V. Dik, and S. B. Dabagov, Nucl. Instrum. Methods Phys. Res., Sect. B 309, 157 (2013).

[26] I. A. Andriyash, E. d'Humières, V. T. Tikhonchuk, and Ph. Balcou, Phys. Rev. ST Accel. Beams 16, 100703 (2013).

[27] A. D. Debus, M. Bussmann, M. Siebold, A. Jochmann, U. Schramm, T. E. Cowan, and R. Sauerbrey, Appl. Phys. B 100, 61 (2010).

[28] K. Steiniger, R. Widera, R. Pausch, A. Debus, M. Bussmann, and U. Schramm, Nucl. Instrum. Methods Phys. Res., Sect. A 740, 147 (2014).

[29] I. A. Andriyash, R. Lehe, and V. Malka, J. Comput. Phys. 282, 397 (2015).

[30] P. Gibbon, Short Pulse Laser Interactions with Matter: An Introduction (Imperial College, London, UK, 2005).

[31] W. Kruer, The Physics of Laser Plasma Interactions (Westview, Boulder, Colorado, USA, 1988).

[32] M. Migliorati, A. Bacci, C. Benedetti, E. Chiadroni, M. Ferrario, A. Mostacci, L. Palumbo, A. R. Rossi, L. Serafini, and P. Antici, Phys. Rev. ST Accel. Beams 16, 011302 (2013).

[33] C. Thaury, E. Guillaume, A. Dopp, R. Lehe, A. Lifschitz, K. Ta Phuoc, J. Gautier, J.-P. Goddet, A. Tafzi, A. Flacco, F. Tissandier, S. Sebban, A. Rousse, and V. Malka, Nat. Commun. 6, 6860 (2015).

[34] A. Arnold and J. Teichert, Phys. Rev. ST Accel. Beams 14, 024801 (2011). 